(2) Open Access Full Text Article

METHODOLOGY

\title{
Customized epithelial debridement for thin ectatic corneas undergoing corneal cross-linking: epithelial island cross-linking technique
}

This article was published in the following Dove Press journal:

Clinical Ophthalmology

17 July 2014

Number of times this article has been viewed

\section{Cosimo Mazzotta' \\ Vincenzo Ramovecchi ${ }^{2}$ \\ 'Unità Operativa Complessa di Oculistica, Siena University Hospital, Siena, Italy; ${ }^{2}$ Unità Operativa \\ Complessa di Oculistica, San Severino Marche Hospital, San Severino Marche, Italy}

\begin{abstract}
Thin corneas with a minimum corneal thickness less than $400 \mu \mathrm{m}$ after epithelial removal represent a contraindication to standard epithelium-off cross-linking (CXL) treatment due to a significant endothelial cell density decrease and potentiality of permanent haze development. Preoperative swelling of the cornea with hypoosmolar riboflavin solutions broadens the spectrum of CXL indications to thin corneas. However the iatrogenic swelling effect might not be durable throughout the CXL procedure increasing the risk of postoperative complications. The transepithelial CXL technique proposed for thin corneas demonstrated poor clinical results and mid- to long-term keratoconus instability. The epithelial island CXL technique with customized pachymetry-guided epithelial debridement was evaluated by means of in vivo laser scanning confocal microscopy, corneal topography, and clinical examination in a 1-year follow-up, in order to assess if it may be considered an alternative surgical option for keratoconic patients with thin corneas undergoing corneal collagen CXL. According to our clinical and in-vivo micro-morphological results the technique results safe, and efficacious in stabilizing progressive keratoconus and may be considered a valid option in the treatment of thin ectatic corneas alone or in combination with hypoosmolar or dextran-free riboflavin solutions.
\end{abstract}

Keywords: cross-linking, keratoconus, thin corneas, epithelial island, surgical technique

\section{Introduction}

A thin cornea (minimum corneal thickness less than $400 \mu \mathrm{m}$ after epithelial removal and before riboflavin instillation) represents a contraindication to standard epitheliumoff cross-linking (CXL) treatment due to significant endothelial cell density decrease and possibility of permanent haze and visual loss. ${ }^{1}$

According to literature ${ }^{2}$ preoperative swelling of the cornea with hypoosmolar riboflavin solutions safely broadens the spectrum of CXL indications to thin corneas that would otherwise not be eligible for classical CXL treatment, showing a stability of keratoconus 1 year after CXL. ${ }^{3}$ The application of the hypoosmolar riboflavin solution preserved cross-linked corneas from developing permanent stromal scars. ${ }^{3}$ Biomechanical studies, measuring the tensile strength of corneal collagen in postmortem porcine corneas models, demonstrated no statistically significant differences in inter-lamellar cohesive force between standard cross-linked corneas and corneas treated after corneal expansion with hypoosmolar riboflavin solutions. ${ }^{4}$

Some authors demonstrated that the intraoperative thinnest pachymetric measurements decreased significantly (by $75 \mu \mathrm{m})^{5,6}$ after the application of iso-osmolar riboflavin solution for 30 minutes (by $55.22 \pm 3.40 \mu \mathrm{m})^{7}$ and increased significantly after hypoosmolar riboflavin application for 10 minutes (by 59.56 $\pm 29.71 \mu \mathrm{m}$ ). However, it was demonstrated that the artificial swelling effect was transient, and the thinnest
Correspondence: Cosimo Mazzotta Policlinico Le Scotte, Viale Bracci, 8 53100 , Siena, Italy $\mathrm{Tel}+390577356618$

Fax +39057735 6618

Email cgmazzotta@libero.it 
pachymetric readings decreased significantly at the end of hypoosmolar riboflavin application. ${ }^{7}$

According to these observations, the iatrogenic swelling effect of the hypoosmolar riboflavin solution might be shortacting and not durable throughout the ultra violet (UV)-A application in collagen CXL procedure increasing the risk of postoperative corneal scarring and endothelial cells' damage. Other studies ${ }^{8}$ evidenced the limited use of hypotonic riboflavin solutions that transiently increased intraoperative corneal pachymetry, although the final thinnest point remained unchanged, and concluded that to increase endothelial safety, new iso-oncotic solutions would be necessary. ${ }^{9}$ Some authors proposed the transepithelial CXL that, according to its limited penetration, seemed more indicated for patients with thin corneas. ${ }^{10,11}$ From results of our previous work, it can be concluded that transepithelial CXL cannot stabilize keratoconus over the mid- to long-term, especially in younger patients with a severer form of the disease. ${ }^{12}$ The limited efficacy of trans-epithelial CXL in terms of poor biomechanical stability was also demonstrated with noncontact depth-dependent analysis of corneal elastic modulus measured by Brillouin microscopy. ${ }^{13}$

A potential surgical approach for the treatment of thin ectatic corneas (under $400 \mu \mathrm{m}$ of minimum corneal thickness after epithelium removal) based on pachymetry guided epithelial debridement was reported by Kymionis et al with promising results. ${ }^{14}$ Transepithelial CXL showed a limited penetration due to epithelial shield provided by epithelium itself and riboflavin, against the UV-photons. ${ }^{15}$ The molecular mechanism of UV attenuation by the riboflavin soaked epithelium serves as selective apical zonal shield preserving endothelium and allowing the possibility to extend CXL treatment to thin corneas. Removing the epithelium where the cornea is thicker allows a better penetration in the corneal para-central area and periphery ensuring a stronger biomechanical effect compared with transepithelial technique. The epithelial island left in situ protects the thin apical cornea from the UV radiation and its borders provide a refraction of UV-A rays deviating their impact in an intermediate stromal level that is theoretically superior to transepithelial technique, allowing a better biomechanical end, biochemical impact of the treatment. ${ }^{15}$ The purpose of this study is to assess the safety and efficacy of customized epithelium-off corneal collagen CXL technique named epithelial-island cross-linking technique (EI-CXL) in a series of ten keratoconic patients with progressive keratoconus and thin corneas, using an in vivo laser scanning confocal analysis, tomographic, and clinical evaluation in a 1-year follow-up.

\section{Methods}

Ten patients with progressive keratoconus, having an average age of 21 years (range 13-26 years) and thin corneas with an average thinnest point of $384 \mu \mathrm{m}$ (range 368-391 $\mu \mathrm{m}$ ) were enrolled in the treatment protocol. All patients were afflicted with stage III keratoconus according to Krumeich's staging with clinical and instrumental progression in the last 6 months of observation (increased $\mathrm{K}$ average $>1$ Diopter, optical pachymetry difference $>10 \mu \mathrm{m}$, uncorrected distance visual acuity (UDVA) and corrected distance visual acuity (CDVA) worsening of at least of 0.1 decimal equivalent). Patients underwent clinical and instrumental pre-operative evaluation before the procedure and postoperatively at day 3 , and 1, 3, 6, and 12 months follow-up, by corneal topography, tomography, Heidelberg retina tomograph (HRT) in vivo laser scanning confocal microscopy, and endothelial cells' count. Corneal topography performed with the Eye-Top corneal topographer (C.S.O. $\mathrm{Srl}$, Florence. Italy) with a polar grid superimposition and altimetry algorithm allowed a reliable keratoconus apex geometric localization and together with laser scanning confocal microscopic analysis increase the information on corneal microstructure and thickness useful for a correct treatment planning (Figure 1).

Patients also underwent a Scheimpflug analysis (Sirius ${ }^{\text {TM}}$; C.S.O. Srl) to explore posterior corneal surface and corneal pachymetry mapping. An optical pachymetry map should be obtained also by time domain corneal optical coherence tomography (OCT) with a white to white (WTW) measurement capability (Global Pachymetry Map, Visante ${ }^{\text {TM }}$ OCT; Zeiss, Jena, Germany). In vivo HRT II laser scanning confocal analysis (Rostock Cornea Module ${ }^{\text {TM}}$; Heidelberg Engineering, Heidelberg, Germany) was used to analyze epithelium, stromal thicknesses, corneal microstructure, endothelial cell count and to detect the regional and whole postoperative micro-morphological changes induced by the customized epithelial island CXL technique at all corneal layers. All patients completed the follow-up. Statistical analysis was conducted by the Mann-Whitney $U$-test for nonparametric data: UDVA and CDVA; by the paired Student's $t$-test for parametric data: simulated $\mathrm{K}$ average power (K Ave), coma power and optical corneal thickness. Patients were also tested by visual analog scale (VAS) pain scale to assess the treatment's tolerability.

\section{Surgical technique}

The technique in Figure 2 includes the following steps:

1. Topical anesthesia and application of the eyelid speculum. 
A

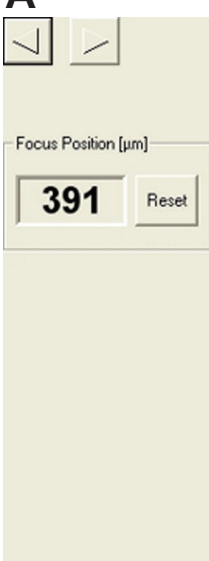

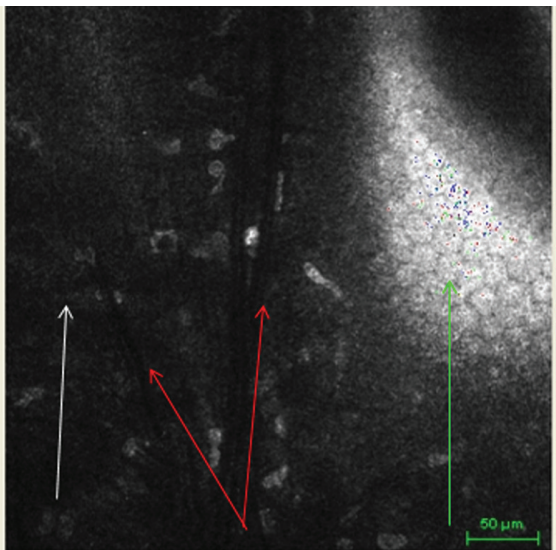

\section{B}

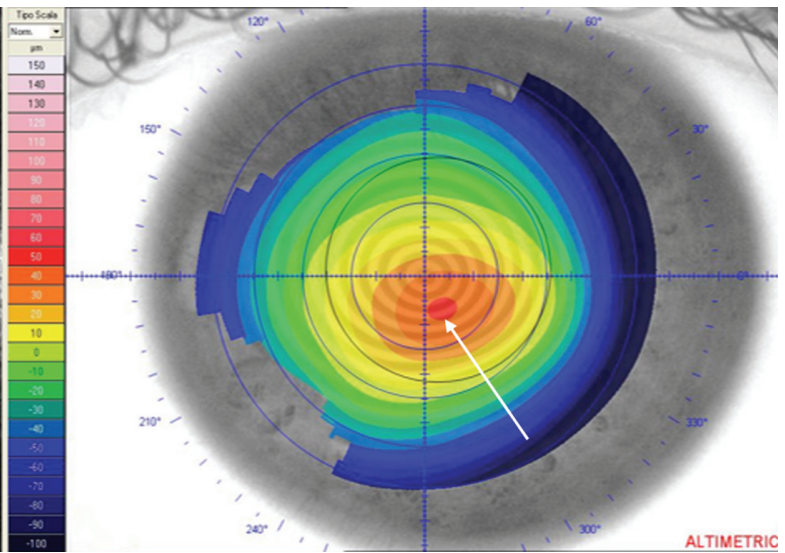

Figure I Confocal pachymetric scan and topographic keratoconus apex localization.

Notes: (A) Confocal scan revealed the typical keratoconus changes of a 39I $\mu \mathrm{m}$ thin cornea: stromal keratocytes (white arrow); Vogt's dark deep striations (red arrows); and pleomorphic endothelium (green arrow). (B) Altimetry map with polar grid superimposition allowing a precise localization of the keratoconus apex in the classic inferiortemporal dislocation (white arrow, red spot).

2. After corneal elevative map and keratoconus area $\left(\mathrm{mm}^{2}\right)$ recognition, the Maloney keratometer can also be used intraoperatively to localize the apical region of keratoconus. Apex region can be marked with a Bores $3.25 \mathrm{~mm}$ optical zone corneal marker. The epithelium must be carefully removed in the para-central and mid peripheral cornea surrounding the epithelial island.

3. Corneal soaking with the conventional riboflavin $0.1 \%$, dextran $20 \%$ for 10 minutes according to Siena University protocol (Dresden modified) avoiding excessive dehydration. ${ }^{6}$ Dextran free riboflavin solution may be used avoiding corneal dehydration. ${ }^{6}$
4. Corneal thicknesses checks corresponding to the epithelial island and in the de-epithelialized area. Corneal thicknesses must be at least $350 \mu \mathrm{m}$ and if not, the corneal stroma should be expanded with 10 minutes of hypotonic $0.1 \%$ riboflavin solution and thickness rechecked.

5. Check of UV-A energy by UV-power meter. The UV-A irradiation is performed with the Caporossi-BaiocchiMazzotta (CBM) Vega X linker (C.S.O. Srl) at $3 \mathrm{~mW} / \mathrm{cm}^{2}$ UV-A emitter for 30 minutes of total UV-A exposure time (six sequential steps of 5 minutes) and energy dose of $5.4 \mathrm{~J} / \mathrm{cm}^{2}$.
A

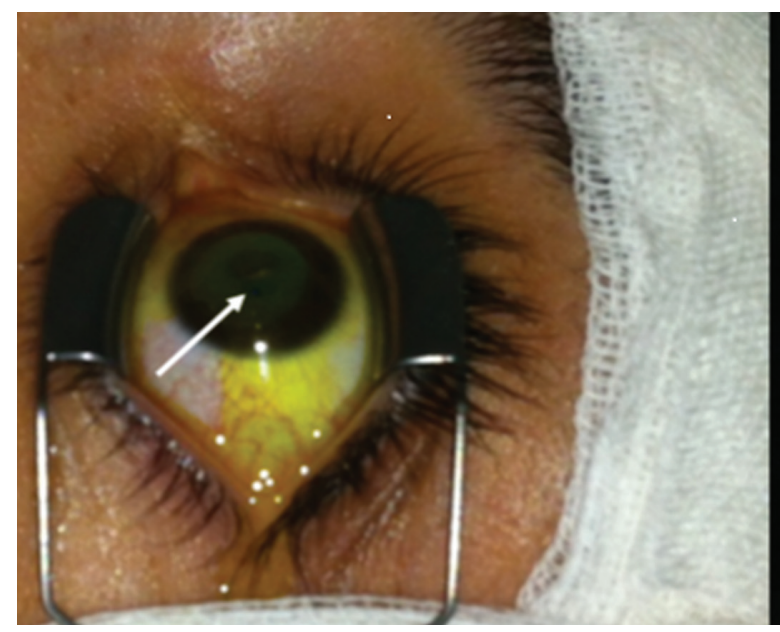

B

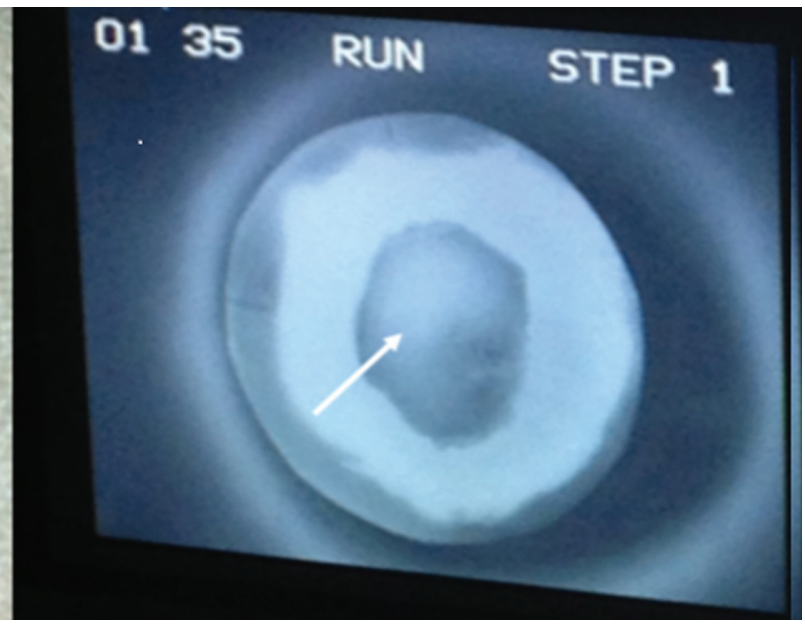

Figure 2 Epithelial island cross-linking technique for thin ectatic corneas.

Notes: Epithelial island (A) white arrow; and $(\mathbf{B})$ white arrow indicating epithelial island during ultra violet-A exposure; the shield effect provided by epithelium in situ is well evident reducing the typical fluorescence of activated riboflavin by the UV-A illumination.

Abbreviation: UV-A, ultra violet-A. 
6. Finally, the cornea is rinsed with a balanced-sterile saline (BSS) solution, covered with a therapeutic soft contact lens (Shalcon Laboratories, Rome, Italy) for 3 days and medicated with ciprofloxacin, diclofenac and sodium hyaluronic acid $0.2 \%$ eye drops four times/day.

\section{Results}

Early post-operative days were comfortable for all treated patients. Therapeutic soft contact lenses were removed after 3 days without epithelial defects or delayed reepithelialization. In vivo laser scanning confocal microscopic (IVCM) analysis performed 1 month after treatment (Figure 3), revealed keratocytes' apoptosis associated with nerve fibers' loss and lacunar edema under the epithelial island at an average depth of $160 \mu \mathrm{m}$ (range 130-180 $\mu \mathrm{m}$ ); keratocytes' apoptosis in the paracentral and de-epithelialized ring was recorded at $250 \mu \mathrm{m}$ depth on average $(230-270 \mu \mathrm{m})$. No change in keratocytes' density was detected in the peripheral area with epithelium in situ. Preoperative endothelial cell density was
$2,451 \mathrm{cells} / \mathrm{mm}^{2}$ on average (range $2,092-3,016$ cells $/ \mathrm{mm}^{2}$ ). No microstructural adverse events were recorded at the endothelial layer during the follow-up and the final endothelial cells' count was 2,456 cells $/ \mathrm{mm}^{2}$ (range 2,062-3,012 cells $/ \mathrm{mm}^{2}$ ).

UDVA and CDVA at 1 month resembled the baseline average values of 0.3 and 0.5 decimal equivalents respectively, demonstrating a not statistically significant improvement of 1 decimal equivalent on average, starting after the 3rd postoperative month, being stable at 1-year follow-up (Figure 4).

Simulated K Ave values in Figure 5A, demonstrated a statistically insignificant decrease of 0.6 Diopters at 1-year follow-up. Coma values in Figure 5B showed a tendency to improve, even though not statistically significant, by a mean value of $-0.8 \mu \mathrm{m}$. Optical pachymetry measurements in Figure 5C, demonstrated a slight, not statistically significant decrease in the first 3 months resembling the baseline average value of $384 \mu \mathrm{m}$, at 6 and 12 months follow-up.

A synopsis of functional parameters and corneal thicknesses is reported in Table 1.

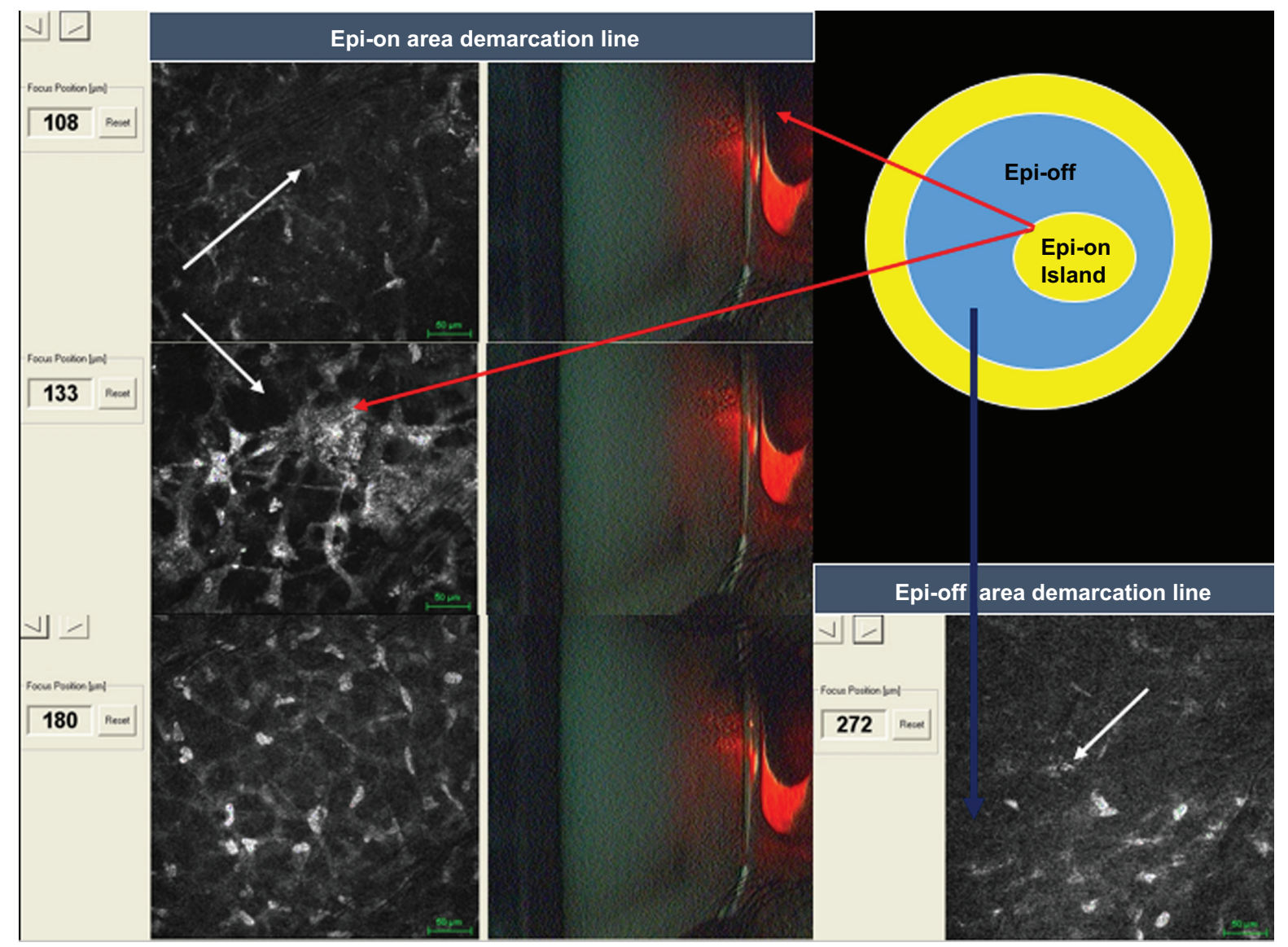

Figure 3 In vivo scanning laser confocal microscopy after epithelial island cross-linking in thin corneas under $400 \mu \mathrm{m}$.

Notes: Red arrows indicate keratocytes apoptosis' and a demarcation line at a depth between I30 and I80 $\mu \mathrm{m}$ (mean I60 $\mu \mathrm{m}$ ) under the epithelial island thinnest point location. White arrows indicate lacunar corneal edema associated with keratocytes' apoptotic bodies. The blue arrow shows the deep demarcation line at $272 \mu \mathrm{m}$ in the epithelium-off paracentral area.

Abbreviations: Epi-on, epithelium-on; Epi-off, epithelium-off. 


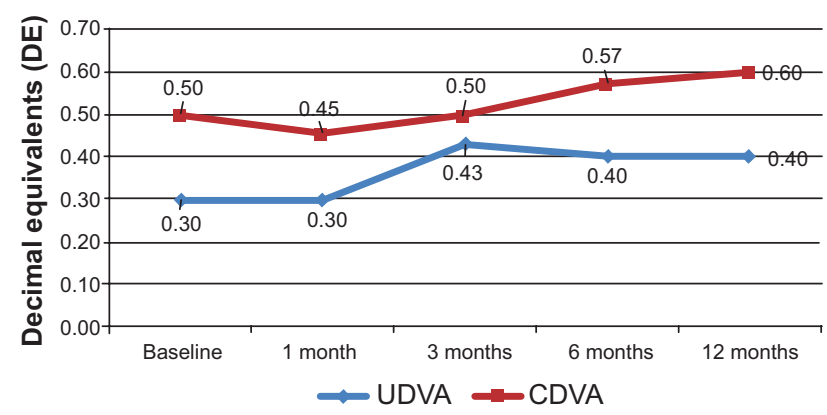

Figure 4 Graphic of uncorrected and corrected distance visual acuity after epithelial island cross-linking technique.

Note: Graphic shows a statistically insignificant improvement of an equivalent of I decimal on average, starting after postoperative month 3, being stable at I-year follow-up.

Abbreviations: UDVA, uncorrected distance visual acuity; CDVA, corrected distance visual acuity.

According to the VAS pain scale, the average value reported in our series was 2 (range 1-3: a mild pain that can be tolerated).

\section{Conclusion}

Epithelial island CXL may represent an additional surgical development in the treatment of ectatic thin corneas. In the authors' experience, it represents a safe and more efficacious alternative to transepithelial CXL due to its limited penetration, biomechanical and clinical efficacy.

EI-CXL surgical approach may be used as an alternative or in combination with corneal expansion by hypoosmolar solutions in order to increase the safety margins of the treatment, ensuring an efficient protection of corneal endothelium.

The epithelial island together with an intrastromal riboflavin shield creates a safe barrier for corneal endothelium corresponding to the corneal thinnest point area. Conversely, the peripheral ring of exposed stroma allowed an adequate riboflavin penetration that could be verified directly at operation microscope obtaining a diffuse yellowish coloration of the cornea and a yellow flare in the anterior chamber of the eye. Removing the epithelium where the cornea is thicker allows a better penetration in the paracentral area and corneal periphery ensuring a stronger biomechanical effect compared with transepithelial technique. The epithelial island left in situ protects the thin apical cornea from the UV radiation, and its borders provide a refraction of UV-A rays deviating their impact in an intermediate stromal level that is theoretically superior to transepithelial technique, allowing a better biomechanical and biochemical impact of the treatment and protecting endothelium.
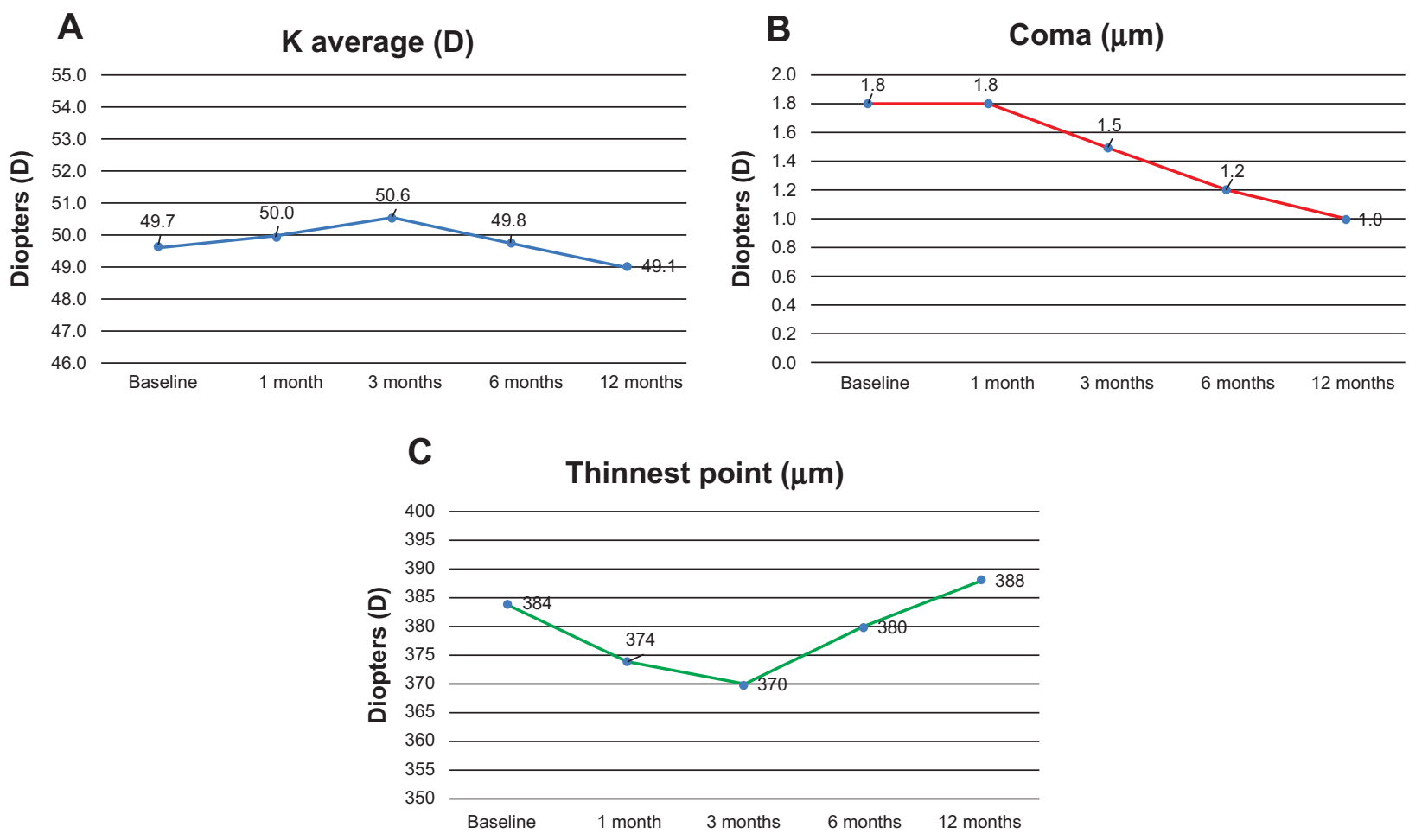

Figure 5 Graphic of simulated $\mathrm{K}$ average values.

Notes: (A) After epithelial island cross-linking technique demonstrated a statistically insignificant decrease of -0.6 Diopters (D) at I-year follow-up. Coma values (B) showed a tendency to improve, even though not statistically significant by a mean value of $-0.8 \mu \mathrm{m}$. Optical pachymetry measurements (C) demonstrated a slight statistically insignificant decrease in the first 3 months resembling the baseline average value at 12 months. 
Table I Functional parameters and optical thickness measurements

\begin{tabular}{llllll}
\hline & Baseline & I month & 3 months & 6 months & I2 months \\
\hline UDVA (decimal equivalent) & 0.30 & 0.30 & 0.43 & 0.40 & 0.40 \\
CDVA (decimal equivalent) & 0.50 & 0.45 & 0.50 & 0.57 & 0.60 \\
K average (Diopters) & 49.7 & 50.0 & 50.6 & 49.8 & 49.1 \\
Coma $(\mu \mathrm{m})$ & 1.8 & 1.8 & 1.5 & 1.2 & 1.0 \\
Thinnest point $(\mu \mathrm{m})$ & 384 & 374 & 370 & 380 & 388 \\
\hline
\end{tabular}

Abbreviations: UDVA, uncorrected distance visual acuity; CDVA, corrected distance visual acuity.

According to micro-morphological outcomes recorded at IVCM, the epithelial island CXL technique enables to overcoming of the pachymetric limitation parameter (over $400 \mu \mathrm{m})$ that is necessary for the safety of the CXL procedure, without affecting the overall benefits of the CXL treatment, as demonstrated in the study results. We did not observe any intra- or postoperative adverse events during the procedure, nor during the entire postoperative follow-up. A not statistically significant reduction in endothelial cells' count with regards to physiologic reduction was observed, namely an average of $2 \% 1$ year after treatment. Patients felt comfortable and according to VAS pain scale tests the early postoperative days were generally painless.

UDVA and CDVA improved by an average value of 1 decimal equivalent. No statistically significant differences were recorded in K Ave and corneal thickness values. Coma aberration showed a slight reduction compared with baseline value. Stabilization of the corneal ectasia was achieved in all treated patients after a 1-year follow-up. The technique proved to be easy to perform without any intraoperative or postoperative complications.

The IVCM study of the cornea performed for the first time in this method of treatment demonstrated the zonal treatment's penetration, revealing the keratocytes' apoptosis under the epithelial island at $150 \mu \mathrm{m}$ of depth on average (range 130-170 $\mu \mathrm{m}$ ). Moreover the depth of keratocytes' apoptosis in the epithelium-off paracentral area surrounding the epithelial island was superimposable (averaging $250 \mu \mathrm{m}$ ) to the standard keratocytes' apoptosis recorded after the conventional epithelium-off CXL treatment, providing a stronger biochemical and biomechanical effect of the procedure that is mandatory for long-term ectasia stabilization.

The study confirms the safety and efficacy of this personalized CXL technique in stabilizing keratoconus in thin corneas with a minimum stromal thickness less than $400 \mu \mathrm{m}$. Even though this study was conducted in a small series of patients who completed the 1 year follow-up, at this point in time a large number of patients have been treated successfully with this simple and less invasive approach.
A larger cohort of patients and more long-term follow-ups are needed to evaluate whether this technique may become a standard treatment option for keratoconus and secondary corneal ectasias in thin corneas, with minimum corneal thickness less than $400 \mu \mathrm{m}$, after epithelial removal, alone or in combination with hypoosmolar or hypo-oncotic dextranfree solutions, avoiding the intra-operative corneal thinning caused by corneal dehydration, reported in literature ${ }^{6}$ where dextran solutions are used.

\section{Disclosure}

The authors report no conflicts of interest in this work.

\section{References}

1. Kymionis GD, Portaliou DM, Diakonis VF, Kounis GA, Panagopoulou SI, Grentzelos MA. Corneal collagen cross-linking with riboflavin and ultraviolet-A irradiation in patients with thin corneas. Am JOphthalmol. 2012;153(1):24-28.

2. Hafezi F, Mrochen M, Iseli HP, Seiler T. Collagen cross-linking with ultraviolet-A and hypoosmolar riboflavin solution in thin corneas. J Cataract Refract Surg. 2009;35(4):621-624.

3. Raiskup F, Spoerl E. Corneal cross-linking with hypo-osmolar riboflavin solution in thin keratoconic corneas. Am J Ophthalmol. 2011;152(1): 28-32.

4. Wollensak G, Spörl E, Mazzotta C, Kalinski T, Sel S. Interlamellar cohesion after corneal cross-linking using riboflavin and ultraviolet A light. Br J Ophthalmol. 2011;95(6):876-880.

5. Kymionis GD, Kounis GA, Portaliou DM, et al. Intraoperative pachymetric measurements during corneal collagen cross-linking with riboflavin and ultraviolet A irradiation. Ophthalmology. 2009;116(12): 2336-2339.

6. Mazzotta C, Caragiuli S. Intraoperative corneal thickness measurement by optical coherence tomography in keratoconic patients undergoing corneal collagen cross-linking. Am J Ophthalmol. 2014;157(6):1156-1162.

7. Kaya V, Utine CA, Y1lmaz ÖF. Intraoperative corneal thickness measurements during corneal collagen cross-linking with hypoosmolar riboflavin solution in thin corneas. Cornea. 2012;31(5):486-490.

8. Chow VW, Biswas S, Yu M, Wong VW, Jhanji V. Intraoperative pachymetry using spectral-domain optical coherence tomography during acelerated corneal collagen cross-linking. Biomed Res Int. Epub 2013 Jul 25.

9. Schmidinger G, Pachala M, Prager F. Pachymetry changes during corneal cross-linking: effect of closed eyelids and hypotonic riboflavin solution. J Cataract Refract Surg. 2013;39(8):1179-1183.

10. Leccisotti A, Islam T. Transepithelial corneal collagen cross-linking in keratoconus. J Refract Surg. 2010;26:942-948.

11. Koppen C, Wouters K, Mathysen D, Rozema J, Tassignon M-J. Refractive and topographic results of benzalkonium chloride assisted transepithelial cross-linking. J Cataract Refract Surg. 2012;38:1000-1005. 
12. Caporossi A, Mazzotta C, Paradiso AL, Baiocchi S, Marigliani D, Caporossi T. Trans-epithelial corneal collagen cross-linking for progressive keratoconus: 24 months clinical results. J Cataract Refract Surg. 2013;39(8):1157-1163.

13. Scarcelli G, Kling S, Quijano E, Pineda R, Marcos S, Yun SH. Brillouin microscopy of collagen cross-linking: noncontact depth-dependent analysis of corneal elastic modulus. Invest Ophthalmol Vis Sci. 2013;54: 1418-1425.
14. Kymionis GD, Diakonis VF, Coskunseven E, Jankov M, Yoo SH, Pallikaris IG. Customized pachymetric guided epithelial debridement for corneal collagen cross-linking. BMC Ophthalmol. 2009;28(9):10.

15. Caporossi A, Mazzotta C, Baiocchi S, Caporossi T, Paradiso AL. Transepithelial corneal collagen cross-linking for keratoconus: qualitative investigation by in vivo HRT II confocal analysis. Eur J Ophthalmol. 2012;22(7):81-88

\section{Publish your work in this journal}

Clinical Ophthalmology is an international, peer-reviewed journal covering all subspecialties within ophthalmology. Key topics include: Optometry; Visual science; Pharmacology and drug therapy in eye diseases; Basic Sciences; Primary and Secondary eye care; Patien Safety and Quality of Care Improvements. This journal is indexed on

Submit your manuscript here: http://www.dovepress.com/clinical-ophthalmology-journal

\section{Dovepress}

PubMed Central and CAS, and is the official journal of The Society of Clinical Ophthalmology (SCO). The manuscript management system is completely online and includes a very quick and fair peer-review system, which is all easy to use. Visit http://www.dovepress.com/ testimonials.php to read real quotes from published authors. 